\title{
Milieu innovateur et entrepreneuriat innovant : la force des proximités et des réseaux
}

\section{Innovative Milieu and Innovative Entrepreneurship: The Power of Proximities and Networks}

\author{
Corinne Tanguy ${ }^{1}$, Dimitri Uzunidis ${ }^{2}$ \\ ${ }^{1}$ Université Bourgogne Franche-Comté, UMR1041 CESAER, INRA, AgroSup Dijon, Réseau de Recherche sur l'Innovation, \\ corinne.tanguy@dijon.inra.fr \\ ${ }^{2}$ Laboratoire de Recherche sur l'Industrie et l'Innovation, Université du Littoral Côte d'Opale, Réseau de Recherche sur \\ l'Innovation, dimitri.uzunidis@univ-littoral.fr
}

RÉSUMÉ. De nombreux travaux se développent aujourd'hui autour de la relation entre l'entrepreneur et le territoire, l'hypothèse sous-jacente étant celle d'un accès plus ou moins aisé selon les territoires aux compétences, connaissances, sources de financement et infrastructures. Le milieu innovateur, défini comme un ensemble d'acteurs (entreprises, institutions, etc.) localisé et ancré dans un territoire dans lequel les interactions se développent de manière multilatérale, constituerait ainsi un environnement favorisant l'apparition de différentes formes d'innovations ainsi que l'émergence de nouvelles entreprises. Cependant le rôle de l'entrepreneur est fondamental dans la concrétisation des opportunités et ressources offertes par un milieu innovateur. En effet c'est lui qui tire parti des ressources du territoire et contribue à la création de technologies nouvelles et d'emplois grâce aux réseaux et proximités qu'il est capable de déployer. En France, comme nous le verrons, la politique des pôles de compétitivité se présente comme une politique incitative en faveur des collaborations et des innovations, et propice à l'entrepreneuriat innovateur.

ABSTRACT. A lot of research is being developed today around the relationship between the entrepreneur and their territory, with the underlying assumption of a more or less easy access to skills, knowledge, funding and infrastructure within a territory. The innovative milieu, defined as a set of actors (companies, institutions, etc.) located and anchored in a territory in which the interactions are developed multilaterally, constitute an environment which favours the appearance of various forms of innovation and the emergence of new companies. However the role of the entrepreneur is fundamental in exploiting the opportunities and resources offered by an innovative milieu. Indeed, he is the person who takes advantage of the territory's resources and contributes to the creation of new technologies and jobs through networks and proximities that he is able to exploit. In France, as we will see, the policy of clusters is presented as an incentive policy for collaboration and innovation, and conducive to innovative entrepreneurship.

MOTS-CLÉS. entrepreneur, milieu innovateur, proximité(s), réseaux, innovation

KEYWORDS. entrepreneur, innovative milieu, proximity(ies), networks, innovation

\section{Introduction}

L'innovation technologique est le moyen le plus sûr pour reconstituer, transformer et étendre les marchés. La croissance des entreprises et la mondialisation accentuée des marchés ont révélé l'importance de poches locales de ressources productives. La proximité géographique entre science, technologie, industrie et finance contribue à l'émergence d'innovations. Les interactions sont organisées par le jeu combiné des acteurs privés et des institutions publiques. Alors que pendant très longtemps le territoire a été considéré en économie comme une variable d'ajustement sans existence propre, les économistes se penchent à l'heure actuelle sur "l'économie locale», comme niveau géographique et économique d'organisation de la production, et, par conséquent, d'émergence de nouvelles activités, de nouveaux biens et services, de nouveaux emplois, de nouveaux revenus, etc. Depuis une quarantaine d'années, le concept de milieu innovateur et l'approche de l'innovation fondée sur la proximité démontrent toujours un peu plus, et mieux, leur pertinence en tant que forme de modélisation de la croissance économique décentralisée, mais aussi d'enrichissement des compétences technologiques des entreprises, y compris internationales. Géographie de l'innovation, districts technologiques, milieux innovateurs, clusters d'innovation, technopoles... les termes sont nombreux, correspondant souvent à des visions différentes 
du processus d'innovation et de son inscription spatiale ${ }^{1}$. Mais au-delà de leurs divergences, ces approches présentent un point commun : elles considèrent que l'innovation peut posséder une dimension spatiale forte et que les firmes retirent un avantage à se localiser dans le même espace (Audretsch, Feldman, 1996). La proximité géographique constitue alors le ferment principal de regroupement des firmes au niveau régional ou local. Ces économistes ne s'inscrivent pas dans un schéma purement linéaire de l'innovation (qui correspondrait à l'idée selon laquelle à l'augmentation des inputs - ici les dépenses de R\&D répondrait l'augmentation des outputs - ici les innovations) mais s'inscrivent davantage dans une vision interactive, qui insiste sur l'importance des réseaux d'acteurs, publics et privés, à un niveau - le territoire - reconnu comme pertinent pour l'établissement d'une politique d'innovation.

De nombreux travaux se développent aujourd'hui autour de la relation entre l'entrepreneur et le territoire, l'hypothèse sous-jacente étant celle d'un accès plus ou moins aisé selon les territoires aux compétences, connaissances, sources de financement et infrastructures. Si le milieu innovateur contribue ainsi à la performance innovante des entreprises par l'offre des ressources scientifiques et techniques qu'il peut organiser, au-delà des relations systémiques, le rôle des acteurs économiques ne doit pas être négligé. David Audretsch et al. (Audrestsch et al., 2006) conçoivent ainsi l'entrepreneur comme le « chainon manquant » entre les politiques publiques visant à favoriser l'innovation et la création d'entreprises innovantes. Nous retrouvons ainsi l'image de l'entrepreneur en tant que relais, comme l'avait décrit J.-B. Say, entre le savant qui produit la connaissance et l'ouvrier qui l'applique à l'industrie. Dans le même ordre d'idées, l'entrepreneur est également conçu comme le créateur non intentionnel de réseaux d'externalités favorisant la création l'épanouissement d'idées nouvelles et de nouveaux marchés (Minniti, 2005).

Quels sont alors les enjeux pour un entrepreneur à s'installer sur un territoire donné? Pour tenter de répondre à cette question, nous présenterons dans une première partie le concept de milieu innovateur ainsi que les proximités en jeu dans les processus d'innovation territorialisés. Nous verrons dans une seconde partie que le rôle de l'entrepreneur est fondamental dans la concrétisation des opportunités et ressources offertes par un milieu innovateur, en particulier grâce aux réseaux qu'il est capable de déployer. Nous terminerons par un point consacré aux politiques mises en place récemment en France et destinées à promouvoir la collaboration et le développement de projets d'innovation sur le territoire et enfin, nous conclurons.

\section{Milieu innovateur et proximites}

L'analyse en termes de système territorialisé d'innovation ou milieu innovateur est relativement récente. Schumpeter a mis très tôt l'accent sur l'apparition en grappe des innovations ou sur les regroupements d'entrepreneurs innovateurs mais la question de l'espace n'était pas présente dans ses analyses, et Perroux (1964), dans son analyse des pôles de croissance s'est focalisé sur le rôle des grandes entreprises en mesure d'entraîner la croissance de leur environnement économique. Il faut attendre les travaux des économistes évolutionnistes dans les années 1980 (Dosi et al., 1988) avec la notion de système national d'innovation pour mettre l'accent sur l'importance des règles, lois et politiques économiques différentes selon les espaces et réellement prendre en compte cette inscription des processus d'innovation dans le territoire.

\subsection{La notion de milieu innovateur}

L'économie locale (ou système productif local) peut être définie comme un espace géographique formé en tant qu'ensemble de rapports systémiques entre entreprises, ainsi qu'entre entreprises, Etat et collectivités territoriales ; ces rapports systémiques caractérisent l'espace localisé par un certain type d'activités et de productions finales. A cette économie, observée et étudiée comme un nœud de relations

1. Pour une présentation des différents types de systèmes territoriaux d'innovation, voir Torre et Tanguy (2014). 
productives, susceptibles, en outre, de contribuer à l'aménagement du territoire (resté, lui, national), les économistes attribuent des qualificatifs montrant la dynamique des rapports combinatoires et complémentaires entre entreprises de différentes tailles au niveau local : « système productif local », " milieu innovateur ».

A la base, c'est le GREMI (Groupe de Recherches Européen sur les Milieux Innovateurs: équipe de chercheurs appartenant à l'Institut de recherches économiques et sociales de l'Université de Neuchâtel, Suisse), qui en 1985, va partir de l'hypothèse que ce sont les milieux régionaux qui sécrètent les différentes formes d'innovations (innovations-produits, innovations-process, innovations organisationnelles, innovations sociales, innovations de formation/qualification, etc.) (Maillat, Perrin, 1992). Un milieu innovateur est défini comme un ensemble d'acteurs (entreprises, institutions, etc.) territorialisé, c'est-à-dire localisé et ancré dans un territoire, ensemble dans lequel les interactions se développent de manière multilatérale (Aydalot, 1986). Elles sont génératrices d'externalités spécifiques à l'innovation et se caractérisent par la convergence des apprentissages vers des formes de plus en plus performantes de gestion en commun des ressources. Les territoires se différencient ainsi en fonction des savoir-faire et ressources spécifiques qu'ils construisent historiquement. L'explication de l'émergence d'une région « à succès » vient du fait que c'est d'abord cette dernière qui a su gérer sa propre capacité à développer de nouveaux produits, de nouvelles techniques et de nouvelles organisations. Cette hypothèse, fondatrice dans la science régionale, va remettre quelque peu en cause les théories économiques traditionnelles qui, au contraire, prônaient jusqu'alors un progrès et une croissance dont les facteurs trouvent principalement leur justification et leur origine à «l'extérieur » (ce sont les modèles dits de « développement par le haut $\gg)$.

A. Marshall (1919) avait démontré que l'efficacité territoriale de ce mode d'organisation tient dans ce que l'on nomme, aujourd'hui, les économies sur les coûts de transaction. La concentration sur un même lieu géographique des principaux acteurs d'un même système productif (essentiellement les producteurs d'un côté, et les utilisateurs de l'autre) permet de faciliter non seulement les transactions, mais aussi les relations réciproques de connaissance et de confiance entre les différents partenaires. La formation et l'accumulation des compétences vont alors former, ce que A. Marshall va appeler, une « atmosphère industrielle » propice à conditionner le marché local du travail. Ce phénomène, l'atmosphère industrielle, est relié à la fois à la compétence et à l'expérience professionnelle des travailleurs et aussi à la localisation des entreprises sur un même territoire. Dans le sens marshallien du district industriel, le milieu réunit une densité de population aux compétences avérées, un ensemble d'acteurs composant les différents maillons d'un même système productif, et enfin, un savoir-faire s'inscrivant fortement dans l'expérience constituée. La caractéristique principale est l'organisation territoriale de la production non pas selon un principe hiérarchique comme une entreprise, mais plutôt selon un principe de collaboration et de coopération entre les différentes unités productives. Ainsi, la notion de solidarité entre les acteurs économiques est très importante. Le système productif local se caractérise surtout par la proximité d'unités productives (entreprises individuelles, de services, centres de recherches et de formation...). Ces unités entretiennent entre elles des rapports d'intensité variable pouvant prendre des formes très différentes : rapports formels ou informels, marchands ou non marchands... Ces alliances portent principalement sur des flux de matières, de services, de main-d'œuvre, de technologies, et de connaissances.

L'hypothèse théorique centrale du concept de milieu innovateur, c'est-à-dire l'environnement socioéconomique d'un territoire forgé par l'histoire (« dépendance de chemin »), est que celui-ci est le produit d'interactions de firmes, d'institutions et de main-d'œuvre. Ces interactions sont obligatoirement le résultat de synergies réciproques (réseaux, maillages, partenariats, etc.) entre les différents agents locaux (publics ou privés) du développement économique et industriel (Garofoli, 1993). Nous pouvons citer ici, comme exemple, les formes de coopération entre les entreprises et les laboratoires de recherche. En fin de compte, c'est d'abord le milieu socio-économique, industriel et scientifique proximal qui participe à la création d'activités nouvelles (notamment à travers l'entrepreneuriat et l'essaimage) et à la genèse des innovations (voir aussi Konstadakopoulos, 2003). Et cela ne pourra «naturellement » se faire que 
si certaines conditions sont respectées. Parmi celles-ci : l'existence, localement, d'un collectif d'acteurs (entreprises, centres de recherches et de formation, administrations publiques, compétences professionnelles...) ; l'existence de ressources matérielles, humaines, financières, technologiques et informationnelles géographiquement proches ; l'existence de savoir-faire spécifiques permettant une activité productive de qualité; l'existence d'un capital relationnel propice à la constitution de réseaux locaux, nationaux ou internationaux (Krugman, 1993) ; et enfin, l'existence de normes, règles et valeurs qui déterminent favorablement le comportement des acteurs économiques.

Ainsi, deux dimensions apparaissent particulièrement constitutives du milieu innovateur : l'innovation technologique, les apprentissages, les savoir-faire et ressources historiquement construits comme principe de compétitivité ; les réseaux comme principe organisationnel. Les réseaux d'innovation dans les travaux du GREMI reposent avant tout sur des relations de proximité géographique. Le milieu innovateur constitue le contexte approprié à leur formation et à leur développement car les agents y partagent des représentations semblables, identifient rapidement des partenaires en leur sein, échangent des informations, se font confiance. Le milieu participe à la constitution des réseaux d'innovation et intervient dans leur dynamique. Réciproquement, alors que les réseaux d'innovation sont par définition a-spatiaux, ils enrichissent le milieu et contribuent à accroître les capacités créatrices de celui-ci (Maillat et al., 1993).

\subsection{Les proximités : de l'espace à l'organisation}

La notion de proximité se trouve largement mobilisée aujourd'hui, autant en économie industrielle qu'en économie de l'innovation (voir entre autres A. Rallet et A. Torre, 2002 ; Bouba-Olga et al., 2008 ; Martin et Boschma, 2010). Mais la polysémie du terme, tout comme la variété et l'étendue de ses applications ( «économies de la proximité ») implique d'en faire un usage raisonné. La proximité considérée $a$ priori se présente comme liée à l'existence d'externalités localisées qui produisent des effets d'agglomération spatiale et des dynamiques territoriales. A cette première signification de proximité physique, se sont rapidement ajoutées d'autres acceptions (recensées par Sierra, 1997) où le champ dans lequel opère la proximité (espace, organisation ou institution) se trouve mêlé au contenu du lien de proximité (information, apprentissage, connaissance ou savoir, technologie, etc.). Sans entrer ici dans une discussion critique qui nous conduirait trop loin, il nous semble que par souci de cohérence il faut accorder la priorité au champ où se joue la relation de proximité (avant de lui donner un contenu). De ce point de vue, deux types de proximité sont généralement considérés lorsque la question de la localisation est couplée avec celle de l'organisation : la proximité spatiale, ou plutôt spatio-temporelle, et la proximité organisationnelle. Ainsi, au-delà de la proximité spatiale, le métier en commun, les codes et normes communes, la proximité organisationnelle, jouent un rôle primordial dans la mise en réseau des entreprises, des centres de recherche et des institutions publiques (Torre, Rallet, 2005).

La spécificité des activités impliquées dans la production de connaissances nouvelles, et des interactions qui leur sont associées, conduit les économistes à introduire, outre la proximité spatio-temporelle et la proximité organisationnelle, la notion de proximité cognitive (Nooteboom, 2002; Boschma, 2004). Celle-ci renvoie au partage plus ou moins formalisé d'expériences, représentations, codes, langages, modèles qui résulte de, et facilite dans le même temps, la communication de toute information au sein des organisations. Par nature, la proximité cognitive occupe une place particulière dans les activités de recherche, mais elle est aussi présente, en arrière fond, à travers toutes sortes de flux de communication interne ou externe à la firme. Dans le cas des interactions liées à la recherche industrielle, la proximité cognitive affecte non seulement les interactions internes aux centres de recherche, mais aussi les interactions externes aux centres avec les autres services des entreprises, ainsi que les interactions avec l'environnement (autres laboratoires et partenaires dans la recherche et l'innovation). C'est aussi à ce niveau que l'entrepreneur joue un rôle fondamental en tant que créateur d'externalités. Pour Minniti (2005), ces réseaux d'externalités sont à l'origine de la formation de modèles mentaux qui permettent de limiter l'ambiguité de l'information délivrée par l'environnement social. Plus le nombre d'entrepreneurs est important, plus le croisement des informations est aisé et plus l'ambiguïté des informations disponibles est faible. Le comportement entrepreneurial devient ainsi un comportement type. 
Il nous parait ainsi possible de proposer une approche tridimensionnelle de la proximité, avant de présenter l'importance de la proximité spatiale dans la génération de nouvelles activités et le lancement d'innovations. Le tableau suivant présente les principaux paramètres caractéristiques des différentes catégories de proximité, ainsi que le domaine d'action et le type d'enjeu qui leur sont liés. Il convient de noter ici que les interactions sont le plus souvent multidimensionnelles; elles constituent une combinaison des différentes dimensions parmi lesquelles une dimension principale se manifeste : dans notre cas, celle de l'espace et du temps. La proximité spatiale est caractérisée par la réduction des distances (et du temps) qui séparent physiquement les acteurs économiques ; condition permissive au développement de relations de reconnaissance et d'inter-reconnaissance entre ceux-ci. La proximité organisationnelle est définie par l'appartenance à une même organisation (entreprise, laboratoire de R\&D, université, service à l'intérieur d'une même entreprise ou administration, etc.), à un même réseau (intra-organisationnel et/ou inter-organisationnel) ou, plus largement, à une même « communauté de destin ». La proximité cognitive renvoie à l'adhésion de différents acteurs à une même conception de l'innovation, au même paradigme (technologique et/ou organisationnel), aux mêmes routines, aux mêmes heuristiques, aux mêmes algorithmes de pensée, aux mêmes conventions, aux mêmes traditions, aux mêmes croyances, aux mêmes codes internes, aux mêmes langages et/ou aux mêmes procédures d'apprentissage, de délibération, de prise de décision et de gouvernance. Elle se situe donc au sein même des organisations, des réseaux et des communautés.

\begin{tabular}{|c|c|c|}
\hline Proximité & Paramètres & Domaine d'action et enjeu \\
\hline Spatio-temporelle & Distance versus vitesse & Déplacement : flux \\
\hline $\begin{array}{l}\text { Organisationnelle } \\
\text { intra- et inter- } \\
\text { organisationnelle }\end{array}$ & $\begin{array}{l}\text { Hiérarchie versus marché } \\
\text { Intra-firme versus inter-firme } \\
\text { Vertical versus } \text { Horizontal } \\
\text { Commandement versus contrat }\end{array}$ & $\begin{array}{l}\text { Coordination } \\
\text { Stratégies, actions, routines }\end{array}$ \\
\hline Cognitive & $\begin{array}{l}\text { Code versus Contenu } \\
\text { Contexte versus Compréhension } \\
\text { (conscience }+ \text { interprétation) } \\
\text { Normalité du comportement } \\
\text { entrepreneurial }\end{array}$ & $\begin{array}{l}\text { Communication } \\
\text { Codes, concepts, idées, connaissances }\end{array}$ \\
\hline
\end{tabular}

Tableau. Les trois catégories de proximité

Cependant si l'idée que les systèmes productifs bénéficient d'une concentration spatiale des activités de recherche et d'innovation est aujourd'hui largement admise et que la proximité géographique apparait comme une condition importante de la réussite technologique, nous savons que grâce à des mobilités de plus en plus étendues, les firmes coopèrent dorénavant dans la majorité des cas avec des organisations qui ne sont pas situées dans la même région. Plus un milieu innovateur est ouvert et connecté à des réseaux d'innovation multiples, plus il est susceptible d'enrichir son réservoir de ressources productives et d'accroître ainsi son attractivité et sa capacité à générer des entrepreneurs. Dès lors, on considère que le milieu est innovateur lorsqu'il est capable de s'ouvrir à l'extérieur et d'y recueillir les informations et les ressources spécifiques dont le système de production localisé qui lui est attaché a besoin pour innover (Uzunidis, 2010). Ainsi aujourd'hui, plus que la présence de milieu innovateur, la ville constituerait le lieu où se trouvent les services (banque et finance, comptabilité, assurance, conseil de gestion et juridique, publicité) et hauts niveaux d'infrastructure et d'équipement, essentielles aux activités d'innovation et aux entrepreneurs innovateurs (Huriot, Bourdeau-Lepage, 2009). Enfin la proximité est une condition préalable à l'établissement de réseaux mais elle n'est pas toujours synonyme de meilleures performances pour les acteurs et peut même quelquefois l'entraver : c'est le " paradoxe de la proximité ». Le courant de géographie économique évolutionniste ou 
évolutionnaire (pour une revue complète de ces analyses, voir le Handbook of Evolutionary Economic Geography édité par Ron Martin et Ron Boschma en 2010) suppose que les différentes formes de proximité peuvent conduire à une dépendance de sentier des réseaux d'innovation et dans certains cas, à un verrouillage régional (les termes de path dependance et de lock in étant largement empruntés au Darwinisme et à la théorie évolutionniste de l'innovation). Les liens internes au milieu peuvent ainsi se révéler selon Bathelt et al. (2004) peu favorables au transfert des connaissances ou des innovations quand les relations locales ne reposent que sur des liens faibles (ou local buzz) ne favorisant pas la transmission des connaissances et des innovations. Les échanges avec l'extérieur (global pipeline) peuvent alors se révéler plus avantageux en termes de politiques d'innovation, puisque porteurs d'innovations radicales se transmettant entre firmes situées à distance

\section{Milieu innovateur et entrepreneur}

La création d'entreprises innovantes ou plus traditionnelles est conditionnée comme nous venons de le voir par la richesse du «milieu». Cependant l'entreprise n'est rien sans l'entrepreneur, soit l'agent économique qui prend la décision de créer une entreprise. C'est lui qui tire parti de l'ensemble des ressources du territoire dans lequel il est inséré et contribue à la création de technologies nouvelles et d'emplois.

\subsection{L'entrepreneur innovateur : ressources et réseaux}

Feldman (2001) recense ainsi les facteurs qui traditionnellement dans la littérature sont associés à l'entrepreneuriat : la disponibilité de ressources financières, le support d'un capital social et relationnel, l'existence de services support et d'expertise entrepreneuriale et enfin, la présence d'universités et centres de recherche. Ce qui est favorable à l'entrepreneuriat innovant dans le cadre de l'économie de réseaux est l'existence d'un espace créé par les relations de proximité : infrastructures de transport, de communication, télécommunications, éducation, ingénierie, etc., relations contractuelles, informationnelles, cognitives, de confiance et de coopération, un réseau dense d'entreprises; un dispositif d'aides fiscales et financières (facilité ou difficulté d'accéder aux aides publiques, le niveau de développement du capital-risque, etc.), etc. (Uzunidis, 2010). Dès lors, tous les milieux ne sont pas ou ne deviennent pas innovateurs. Certains sont même franchement inhibiteurs et il est primordial pour un territoire d'assurer le maintien et le renouvellement de ses compétences-clés (Mendez, Mercier, 2006), c'est-à-dire des compétences critiques pour sa survie. Cette question est d'autant plus importante quand ces territoires abritent des secteurs industriels anciens, mais qui doivent pourtant s'insérer dans la concurrence internationale. Or un manque de ressources financières et/ou de soutien public (Depret et al., 2010), ou bien l'existence de conflits et la difficulté à construire et partager un projet commun entre acteurs ( Torre, Beuret, 2012) peuvent ainsi constituer des obstacles aux dynamiques entrepreneuriales. La capacité à entreprendre résulte alors de la variété et de la richesse du potentiel de ressources mobilisé et mobilisable par l'entrepreneur (Uzunidis, 2010). La composition de ce potentiel de ressources dépend de facteurs extérieurs à l'entreprise et à l'entrepreneur (c'est-à-dire de la richesse du milieu innovateur). A leur tour, ce sont les entrepreneurs eux-mêmes qui en s'adaptant et en saisissant de nouvelles opportunités créent les facteurs et conditions qui favorisent leurs affaires et contribuent au développement de ces ressources externes (Feldman, 2001).

Les entrepreneurs, dans un contexte d'innovation, utilisent en particulier leurs réseaux sociaux pour valider l'existence d'une opportunité d'affaires, c'est-à-dire attester de l'existence d'une possibilité de gain économique (Chabaud, Ngijol, 2010). Au-delà des ressources financières initiales permettant d'amorcer le projet, les entrepreneurs sont en effet confrontés à de nombreuses difficultés, surmontées grâce à la mobilisation de ressources relationnelles. En matière de recherche d'informations ou d'acquisition de ressources, les entrepreneurs s'appuient alors essentiellement sur les liens forts car ceux-ci leur apparaissent à la fois plus faciles d'accès et marqués par la confiance (Chabaud, Ngijol, 2010). L'accent est donc mis sur le capital humain des entrepreneurs. Grâce à leur réseau de relations sociales, ils auront accès à des ressources (en connaissances, financières, etc.) et à des informations 
qu'ils ne possèdent pas. L'entrepreneur est encastré dans un réseau de relations sociales (M. Granovetter, 2006). La constitution de réseaux d'entreprises est également un moyen de réduire les risques liés à l'incertitude du marché. Plus les réseaux seront protecteurs, plus l'acte d'entreprendre sera facilité (Uzunidis, 2010). Cependant s'il est toujours préférable de s'appuyer sur les ressources issues de ses relations sociales pour démarrer son activité, celles-ci peuvent devenir un problème, à terme, pour son développement si elle se traduit par des obligations trop lourdes pour l'entreprise (ingérence dans la gestion de l'entreprise, obligation d'embaucher une personne de sa famille ou de faire des « ristournes " à ses proches, etc.) (Granovetter, 2003). Les réseaux sociaux affecte l'activité économique pour trois raisons majeures : (1) ils influencent les flux et la qualité de l'information, (2) ils sont une source importante de récompenses et de sanctions qui ont un impact d'autant plus important qu'ils viennent d'individus personnellement connus, (3) ils créent de la confiance, qui se définit comme que les autres acteurs économiques feront la «chose juste», en dépit d'une claire incitation à faire le contraire. L'action économique est bien encastrée dans des systèmes de relations personnelles et les réseaux individuels ont toujours une part locale, mais l'importance et la nature des liens locaux dans un site déterminé varient selon les mobilités des acteurs et leurs caractéristiques (fréquence, caractère périodique ou non, etc). Ainsi les réseaux localisés d'innovation peuvent s'expliquer moins par la nécessité fonctionnelle du face à face pour échanger des connaissances que parce que la coopération s'établit entre des chercheurs ou des ingénieurs issus de la même université ou appartenant au même réseau social ou familial (Grossetti, Bès, 2003). Autrement dit, les effets de proximité peuvent varier selon les contextes et si les individus étaient parfaitement mobiles, les effets de proximité disparaitraient.

L'entrepreneur est une figure, un concept, une fonction difficiles à cerner par les outils théoriques existants (Tounes, Fayolle, 2006). Les qualités personnelles et la personnalité de l'entrepreneur jouent certainement un rôle important dans la décision de créer ou de reprendre une petite entreprise. Mais la fonction et l'action d'entreprendre sont définies par la dynamique macro-systémique d'accumulation et de profit. D'autre part, l'acte créatif s'inscrit dans un cadre socialisé et le fait est qu' «on ne naît pas entrepreneur, on le devient $»$ : on le devient par la mobilisation de son potentiel de ressources composé de capitaux, de connaissances et de relations (Boutillier, Uzunidis, 2001 et 2006). Le capital pour l'investissement et le fonctionnement; les connaissances pour le choix et la décision; les relations pour le financement, l'association et l'écoulement de la production.

\subsection{Politiques de constitution de milieux innovateurs et d'émergence d'un entrepreneuriat innovant}

L'ancrage territorial de l'entreprise lui permet de se constituer un réservoir de ressources (et parfois un marché) pour amortir les coûts inhérents à ses investissements dans une économie en constante transformation. Mais cet ancrage territorial dépend de la qualité dudit réservoir par rapport aux attentes de l'entreprise en matière d'innovation et d'expansion commerciale. D'où la nécessité pour les gouvernements et les collectivités territoriales de faire système, d'organiser la valorisation des ressources dans le but de faire naître de multiples processus d'innovation tenant compte la concurrence-coopération entre mêmes acteurs en économie ouverte (voir notamment Amable, Barré, Boyer, 1997 ; Pitelis, Sugden, Wilson, 2005). Il s'agit d'un système d'offre de ressources productives susceptible d'engendrer un entrepreneuriat technologique et d'attirer les grandes entreprises ayant des performances affirmées en matière d'innovation (Madeuf, Carré, Lefebvre, Milelli C., 2005 ; Uzunidis, 2004).

Depuis deux décennies les pouvoirs publics et les grandes entreprises ont mis en ouvre un important dispositif d'administration de la création d'entreprise (visant l'augmentation du " potentiel de ressources » de l'entrepreneur), afin de combler les vides provoqués par l'activité économique et pour se doter de moyens d'expérimentation des nouvelles valeurs marchandes et des nouvelles pratiques sociales. Ainsi dans les années 2000, l'Organisation de coopération et de développement économiques (OCDE) incite les gouvernements et les institutions territoriales à intervenir dans l'économie pour "stimuler l'esprit d'entreprise » (OCDE, 1999). Depuis les années 1980, les différents travaux sur les systèmes territorialisés d'innovation (Milieux Innovateurs, Systèmes Productifs Localisés, Clusters) ont donné lieu à la mise 
en œuvre de politiques publiques (Héraud, 2003). Le dispositif des systèmes productifs locaux, soutien logistique et financier à des réseaux sélectionnés de firmes, a été lancé en France par la DATAR en 1998. En 2009 la DATAR a lancé la politique des grappes d'entreprises, concernant des groupes d'entreprises ayant des projets communs au niveau d'un territoire, en particulier dans des secteurs d'activité caractérisés par une faible activité de R\&D. L'exemple le plus frappant de la mise en œuvre d'une politique structurelle d'aide à l'entrepreneuriat et d'attractivité économique des «milieux » est celui de la constitution des pôles de compétitivité (Fontagné, Lorenzi, 2005), un peu partout dans le monde industriel (pour l'Europe, voir Peyroni, 2002 ; Uzunidis, Laperche, 2006) mais aussi dans certains grands pays en développement (l'Inde, la Chine, etc.). La politique des pôles de compétitivité mise en œuvre en France depuis 2004 a ainsi pour objectif de favoriser les projets collaboratifs de recherche et de développement entre acteurs localisés sur un même territoire.

Selon la définition officielle ${ }^{2}$, un pôle de compétitivité est « la combinaison sur un espace géographique donné d'entreprises, de centres de formation et d'unités de recherche publiques ou privées, engagés dans une démarche partenariale destinée à dégager des synergies autour de projets communs au caractère innovant. Ce partenariat s'organise autour d'un marché et d'un domaine technologique et scientifique qui lui est attaché et doit rechercher la masse critique pour atteindre une compétitivité mais aussi une visibilité internationale ».

Les pôles de compétitivité combinent l'attractivité (effets d'agglomération) et la diffusion (effets de dispersion) ; deux facteurs essentiels pour la naissance et la propagation de l'innovation. Ainsi, un territoire construit économiquement par un potentiel d'innovation important, par un système financier diversifié et riche, par un large éventail de services à l'industrie, par un système de protection de la propriété industrielle et de secret, par un tissu dense de PME, par l'accès à un grand marché régional/national, par des politiques incitatives en recherche et en innovation est propice à l'entrepreneuriat innovateur alimentant ainsi l'" économie de réseaux ».

Les petites entreprises innovantes s'introduisent dans des réseaux formés par les grands groupes et coordonnés souvent en rapport avec l'implantation territoriale (Uzunidis, Boutillier, 2003). C'est le cas des parcs scientifiques où les firmes détenant des avantages technologiques et commerciaux de grande envergure créent, elles-mêmes, de petites entreprises spécialisées (de sortes de laboratoires de recherche) gérées par les entrepreneurs-chercheurs. Dans d'autres cas, ce sont les cadres de la grande entreprise qui sont priés par la société mère de créer une entreprise d'expérimentation de technologies nouvelles (intrapreneuriat). Il n'est pas rare non plus de voir l'apparition d'un " entrepreneuriat de spéculation » (Tidd, Bessant, Pavitt, 2005) au profit des grandes entreprises. Il s'agit des personnes très qualifiées qui, aidées par les prêts bonifiés et les subventions étatiques, créent une entreprise pour la revendre à une plus grande après que le produit ou le procédé de production soit mis au point. La connexion des petites entreprises spécialisées avec les groupes est réalisée grâce à une stratégie de veille et financière adaptée à ce sujet. Les sociétés de capital-risque (sociétés de prise de participation dans le capital d'une entreprise qui vient d'être créée), les business angels (individus fortunés qui investissent dans des projets innovants) et autres financiers (les Fonds de pension sont très actifs) engagent les capitaux des épargnants dans les nouvelles entreprises des technologies de l'information et des biotechnologies.

\section{Conclusion}

Aussi bien les aspects locaux qu'entrepreneuriaux de l'innovation révèlent de la mécanique de formation et d'appropriation de tous les ingrédients (capital) de l'activité productive. La question de l'appropriabilité est devenue cruciale pour le fonctionnement (la localisation ou la création) d'une entreprise. L'entreprise a tendance à se servir davantage de son environnement qu'à investir, par exemple, dans toutes les phases de création technologique ; ce qui peut être expliqué par le fait que les

2. Site web du gouvernement, http://www.competitivite.gouv.fr 
investissements dans l'acquisition (appropriation) des ressources de production sont moins coûteux que ceux consacrés à la formation de ces ressources; ce qui explique aussi l'attractivité (en économie ouverte) d'une région disposant des ressources scientifiques et techniques abondantes. La création d'entreprises innovantes ou plus traditionnelles est conditionnée par la richesse du « milieu ». Si les facteurs liés à l'éducation, à l'environnement, aux soins médicaux, à la finance, aux infrastructures, aux logements, etc. agissent sur la trajectoire du coût marginal d'une entreprise ou d'une activité, ils influent aussi sur le rendement des capitaux investis. L'idée alors du «réseau» et du «milieu innovateur » est apparue pour s'imposer dans l'observation et l'analyse économique.

Le concept de milieu innovateur met en exergue un principe fort au niveau des systèmes d'innovation : il renforce l'idée que la capacité d'innovation des entreprises est intimement liée aux facteurs sociaux, économiques et politiques qui les entourent (Crevoisier, 2000). Le « milieu innovateur » désigne alors le plus souvent la capacité d'une économie locale à engendrer des innovations via l'émergence de nouvelles entreprises et la localisation d'anciennes entreprises dans sa zone géographique, là où la valorisation industrielle de la recherche organise la création de petites entreprises innovantes. L'économie locale se présente, ainsi, comme un système territorialisé de valorisation de toutes sortes de capitaux et d'échanges marchands. Bénéficiant d'une certaine autonomie d'organisation (légale et économique) des ressources productives, sa caractéristique première est la formation et la mise en valeur des ressources spécifiques et la réalisation des combinaisons particulières de ces ressources spécifiques (cf. le cas de Sophia-Antipolis étudié par D. Elidrissi et V. Hauch, 2008). Ces dernières sont composées de capital et de travail ayant des formes et des contenus spécifiques par rapport à des activités et à des secteurs donnés : spécifiques sur le plan technologique, financier ou démographique, mais aussi sur le plan des compétences, des qualifications, du niveau d'éducation, etc. L'économie locale devient, alors, un « milieu innovateur », réducteur des risques liés à l'incertitude d'un investissement donné et initiateur de processus d'innovation, y compris grâce à la création d'entreprises et à l'accueil d'entreprises technologiques existantes.

Cependant l'entreprise n'est rien sans l'entrepreneur, soit l'agent économique qui prend la décision de créer une entreprise. C'est lui qui tire parti des ressources du territoire dans lequel il est inséré et qui contribue à la création de technologies nouvelles et d'emplois. L'innovation, comme le suggèrent Audrestch, Keilbach et Lehmann (2006) est un processus sans fin, car les nouveaux créateurs d'entreprises crée une activité nouvelle en valorisant des idées qui ne l'ont pas été par d'autres entrepreneurs. L'investissement de millions d'euros ou de dollars pour financer la R\&D reste stérile en l'absence d'entrepreneurs. De plus, la multiplication d'initiatives individuelles contribue à faire de l'entrepreneur un modèle comportemental que d'autres agents économiques peuvent être amenés à imiter, contribuant du même coup à la création d'innovations nouvelles. Dans le cadre défini d'un territoire particulier, les agents économiques ont créé un réseau de relations sociales (Granovetter, 2006) de toutes natures grâce auquel ils accèdent aux ressources dont ils ont besoin pour mener leur projet à ses fins, y compris les ressources informationnelles.

\section{Références}

AMABLE B., BARRÉ R., BOYER, R., Les systèmes nationaux d'innovation à l'ère de la globalisation, Economica, Paris, 1997.

Audrestch D.B., KeILBACH M. C., LeHMANN E.E., Entrepreneurship and Economic Growth, Oxford University Press, Oxford, 2006.

AUdRETSCH D.B., FELDMAN P.M., R\&D Spillovers and the Geography of Innovation and Production, American Economic Review, vol. 86, p. 630-640, 1996.

AydALOT Ph., Les milieux innovateurs en Europe, Economica / GREMI, Paris, 1986.

BATHELT H., SCHULDT N., Between Luminaires and Meat Grinders: International Trade Fairs as Temporary Clusters, Regional Studies, vol. 42, p. 853-868, 2008.

Boschma R., Proximité et innovation, Économie Rurale, vol. 280, p. 8-24, 2004. 
Bouba-Olga O., CARrinCAZeauX C., CORIS C., La proximité, 15 ans déjà ! Première partie : propositions théoriques, Revue d'Economie Régionale et Urbaine, vol. 3, p. 279-287, 2008.

BOUTILLIER S., UZUNIDIS D., L'utilité marginale de l'entrepreneur, Innovations, vol. 13, n 1, p. 17-42, 2001.

BOUTILLIER S., UzUNIDIS D., L'aventure des entrepreneurs, Studyrama, Paris, 2006.

Chabaud D., NGiJOL J., Quels réseaux sociaux dans la formation de l'opportunité d'affaires ?, Revue française de gestion, vol. 206, p. 129-147, 2010.

CREVOISIER O., L'approche par les milieux innovateurs : état des lieux et perspectives, Revue d'Économie Régionale et Urbaine, vol. 1, p. 153-166, 2000.

Depret M.H., Hamdouch A., Monino J.L., Poncet C., Politiques d'innovation, espace régional et dynamique des territoires : un essai de caractérisation dans le contexte français, Innovations, vol. 33, n 3, p. 85-104, 2010.

Dosi G., Freeman C., Nelson R.R., Silverberg G., Soete L., (eds), Technical Change and Economic Theory, Pinter, London, 1988.

ELIDRISSI D., HAUCH V., Les systèmes régionaux d'innovation : superpositions et articulations. Le cas de Sophia Antipolis, La revue des sciences de gestion, vol. 233, p. 59-66, 2008.

FELDMAN M.P., The Entrepreneurial Event Revisited: Firm Formation in a Regional Context, Industrial and Corporate Change, vol. 10, $\mathrm{n}^{\circ} 4$, p. 861-891, 2001.

Fontagnén L., LORENZI J.-H., Désindustrialisation, délocalisations, Conseil d'analyse économique, La documentation française, Paris, 2005.

GAROFOli G., Economic Development, Organization of Production and Territory, Revue d'Économie Industrielle, vol. 64, p. 22-37, 1993.

Granovetter M., L'influence de la structure sociale sur les activités économiques, Sociologies Pratiques, vol. 13, p. 9-36, 2006.

GRANOVETTER M., La sociologie économique des entreprises et des entrepreneurs, Terrains et travaux, ENS Cachan, vol. 4, p. 167-206, 2003.

GrossetTi M., BÈS, M.-P., Proximité spatiale et relations science - industrie : savoirs tacites ou encastrement (Polanyi ou Polanyi) ? Réflexions à partir d'une étude sur les collaborations CNRS - entreprises en France, Revue d'Economie Régionale et Urbaine, vol. 5, n² 2, p. 777-788, 2003.

HÉRAud J.-A., Régions et innovation, in Mustar P., PenAn H., (dir.), Encyclopédie de l'innovation, Economica, Paris, 2003.

HURIOT J.-M., BouRdEAU-LePAGE L., Economie des villes contemporaines, Economica, Paris, 2009.

KonstadAKOPOUlos D., Milieux innovateurs et apprentissage dans le Sud-Ouest de l'Angleterre, Innovations, vol. 11, p. 139-154, 2000.

KRUGMAN P., First Nature, Second Nature and Metropolitan Location, Journal of Regional Science, vol. 33, p. 129-144, 1993.

MAdeuf B., CARré, G., LefebVRe G., Milelli C., TIC et économie de la proximité : organisation et localisation de la R\&D au sein des entreprises globales, Innovations, vol. 21, p. 243-272, 2005.

MaILlat D., QueVIT M., SenN L., (eds), Réseaux d'innovation et milieux innovateurs : un pari pour le développement régional, GREMI/EDES, Neuchâtel, 1993.

MaILlat D., PERRIN J.-C., (dir.), Entreprises innovatrices et développement territorial, Enquête GREMI II, EDES, Neuchâtel, 1992.

MARSHALL A., Industry and Trade, MacMillan, London, 1919.

MARTIN R., Boschma R., (eds), The Handbook of Evolutionary Economic Geography, Edward Elgar, Cheltenham, 2010.

MENDEZ A., MERCIER D., Compétences-clés de territoires. Le rôle des relations interorganisationnelles, Revue française de gestion, vol. 164, $\mathrm{n}^{\circ}$ 5, p. 253-275, 2006.

MiNNITI M., Entrepreneurship and Network Externalities, Journal of Behavior and Organization, vol. 57, p. 1-27, 2005.

Nooteboom B., A Cognitive Theory of the Firm, Paper for ESNIE Workshop Alternative Theories of the Firm, Paris, November, 2002. 
OCDE (Organisation de la coopération et du développement économiques) (1999), Stimuler l'esprit d'entreprise, OCDE, Paris.

PERrouX F., Industrie et création collective, Tome 1: Saint Simonisme au XXème siècle et création collective, Presses Universitaires de France, Paris, 1964.

Peyroni J., Le schéma de développement de l'espace communautaire, La documentation française, Paris, 2002.

Pitelis C., Sugden R., WiLSON J.R., Clusters and Globalisation, Edward Elgar, Cheltenham, 2005.

RAllet A., TORRE A., Proximité Géographique ou Proximité Organisationnelle ? Une analyse spatiale des coopérations technologiques dans les réseaux localisés d'innovation, Economie Appliquée, p. 147-171, 2002.

SIERRA C., Proximité(s), interactions technologiques et territoriales, Revue d'économie industrielle, vol. 82, p. 7-38, 1997.

TidD J., Bessant J., PAVITT K., Managing Innovation, J. Wiley and Sons, Chichester, 2005.

TORRE A., TANGUY C., Les systèmes territoriaux d'innovation : où en est-on ?, in BouTILLIER S., ForeSt J., GALLAUD D., Laperche B., Tanguy C., Temri L., Principes d'Economie de l'Innovation, Bruxelles, Peter Lang, p. 307-319, 2014.

TORre A., Beuret J.E., Proximités Territoriales, Paris, Economica, 2012.

TORRE A., RAllet A., Proximity and Localization, Regional Studies, vol. 39, n 1, p. 47-59, 2005.

TOUNes A., FAYOlle A., L'odyssée d'un concept et les multiples figures de l'entrepreneur, La revue des sciences de gestion, vol. 220-221, p. 17-30, 2006.

UzUNIDIS D., Milieu innovateur, relations de proximité et entrepreneuriat. Analyse d'une alchimie féconde, Revue canadienne de science régionale, vol. 33, p. 91-106, 2010.

UZUNIDIS D., (dir.), L'innovation et l'économie contemporaine, De Boeck, Bruxelles, 2004.

UzUNIDIS D., BOUTILliER S., L'Etat, les entrepreneurs et la mondialisation, in M. Van Cromphaut, L'Etat à l'ère de la mondialisation, Economie et Innovation, L'Harmattan, Paris, 2003.

UZUNIDIS D., LAPERCHE B., La territorialisation des aides et de la politique européenne de R\&D. Quelles perspectives pour la France?, ORATE, Union Européenne, 2006. 